\title{
FORESTERIE COMMUNAUTAIRE, SAVOIRS AUTOCHTONES ET GOUVERNANCE PARTICIPATIVE AU NICARAGUA
}

Sandrine Fréguin-Gresh

Presses de Sciences Po (P.F.N.S.P.) | « Autrepart »

2017/1 $\mathrm{N}^{\circ} 81$ | pages 41 à 56

ISSN 1278-3986

ISBN 9782724635348

Article disponible en ligne à l'adresse :

https://www.cairn.info/revue-autrepart-2017-1-page-41.htm

\section{Pour citer cet article :}

Sandrine Fréguin-Gresh, « Foresterie communautaire, savoirs autochtones et gouvernance participative au Nicaragua », Autrepart 2017/1 (N 81), p. 41-56. DOI 10.3917/autr.081.0041

Distribution électronique Cairn.info pour Presses de Sciences Po (P.F.N.S.P.).

(C) Presses de Sciences Po (P.F.N.S.P.). Tous droits réservés pour tous pays.

La reproduction ou représentation de cet article, notamment par photocopie, n'est autorisée que dans les limites des conditions générales d'utilisation du site ou, le cas échéant, des conditions générales de la licence souscrite par votre établissement. Toute autre reproduction ou représentation, en tout ou partie, sous quelque forme et de quelque manière que ce soit, est interdite sauf accord préalable et écrit de l'éditeur, en dehors des cas prévus par la législation en vigueur en France. Il est précisé que son stockage dans une base de données est également interdit. 


\title{
Foresterie communautaire, savoirs autochtones et gouvernance participative au Nicaragua
}

\author{
Sandrine Fréguin-Gresh*
}

« Nous travaillons ensemble pour défendre nos forêts, car les menaces que nous subissons peuvent détruire nos succès et anéantir nos aspirations à vivre bien »

Luis Tacio, ex-Président de COOSIPBAA R.L.

Au cours des vingt dernières années, la foresterie communautaire s'est imposée comme une notion clef des projets de développement mis en œuvre dans les régions forestières tropicales [Sabogal et al., 2008 ; Arnold, 1991], visant à permettre d'atteindre les trois objectifs économiques, sociaux et environnementaux d'un développement durable. Cette notion renvoie à une forme de gestion et d'exploitation des forêts dans lesquelles des communautés, c'est-à-dire des individus ayant des droits sur les ressources ${ }^{1}$ et qui élaborent ensemble et appliquent des règles d'accès et d'usage de la forêt, participent à son exploitation [USAID, 2013]. En incluant les populations vivant dans, autour et de l'exploitation des forêts, la foresterie communautaire doit autoriser une gestion des ressources forestières concertée, socialement acceptable et économiquement rentable, ainsi qu'une extraction du bois plus respectueuse de la nature. L'engouement qui s'est opéré depuis les années 1990 pour la foresterie communautaire s'inscrit dans la trajectoire de l'émergence du développement durable et s'est imposé mondialement après le Sommet de la Terre en 1992 [Milol, 2007 ; Le Coq et al., 2013]. En effet, l'essor de la foresterie communautaire s'accompagne d'un recentrage des projets sur les populations locales, notamment les communautés autochtones des régions forestières, qui affirment leurs droits à contrôler l'exploitation des ressources, alors que les arbres sont au cœur de leurs moyens d'existence [Arnold, 1991]. Aujourd'hui, et même si certains s'interrogent sur sa réussite [Cuny, 2011], de nombreux auteurs soulignent que l'introduction de la notion de forêt

\footnotetext{
* Agroéconomiste et géographe, département environnement et sociétés, UMR ART-Dev (acteurs, ressources et territoires en développement), CIRAD.

1. Quels que soient les droits : partiels ou complets ; légalement reconnus ou non ; juridiques, traditionnels ou coutumiers.
} 
communautaire dans des projets de développement a permis d'accroître la participation des populations locales dans des modes d'exploitation commerciale de la forêt, plus respectueuse de l'environnement [Arnold, 1991], tout en affirmant et en confortant leurs droits.

Au Nicaragua, la foresterie communautaire émerge durant les années 2000 et devient l'un des piliers des interventions de développement dans la Côte Atlantique Nord. Comme dans d'autres pays d'Amérique Centrale, la notion s'impose pendant une période compliquée de réconciliation nationale qui fait suite à des années de conflits armés ayant bouleversé la trajectoire sociopolitique et économique du pays et souvent participé à la dynamique de déforestation [Larson, 1989]. L'émergence de la foresterie communautaire dans cette région est favorisée par le contexte national où la manière de penser la gouvernance évolue profondément, mettant en avant l'articulation de l'État avec la société pour une amélioration de la légitimité de la prise de décision et de l'efficacité des interventions [Le Coq et al., 2013]. La reconnaissance des droits des populations autochtones de la Côte Atlantique Nord à se gouverner selon leurs savoirs et leurs normes, permise par la promulgation de la Constitution et le statut d'autonomie de 1987, accompagne cette émergence en même temps que le transfert de pouvoirs vers les niveaux inférieurs de la hiérarchie administrative pour gérer les ressources naturelles [Larson, 2002]. La décentralisation de la gestion environnementale inscrit les savoirs dans les demandes et les intérêts des communautés autochtones au cœur du programme politique et renforce les responsabilités de leurs représentants [Kaimowitz, Faune, Mendoza, 2003]. En conséquence, la reconnaissance des savoirs autochtones dans la manière de concevoir les projets s'impose et les interventions de développement réorientent leurs logiques, de la promotion d'un protectionnisme sanctifiant la nature dans des aires protégées à une nouvelle conception, fondée sur une exploitation forestière associant les communautés. Par cela, la foresterie communautaire vise à conforter l'exercice des droits de ces populations, qui seraient ainsi mieux armées pour lutter contre la pauvreté, tout en conservant la nature dans une logique de compétitivité socialement équitable et acceptable [Jarquín, 2003]. La foresterie communautaire s'impose alors là où la forêt joue de manière ancestrale un rôle social, culturel, et économique central. Pour les acteurs de la coopération technique promouvant ces projets et arguant - au moins sur le papier - de l'importance des savoirs autochtones et de la relation à la nature spécifique de ces populations, une exploitation forestière participative ne peut qu'être respectueuse de l'environnement. Pour les coopérants techniques, il s'agit de les aider à impulser un changement pour mieux les connecter au marché. Cela suppose de les former à « conserver en exploitant ${ }^{2}$ » et à restaurer les ressources forestières, dégradées par l'avancée de la colonisation agricole.

2. Cette citation fait référence à la devise du Centre agronomique tropical de recherche et d'enseignement (CATIE). 
Au plan national, la foresterie communautaire est envisagée comme « la reconnaissance de l'exercice effectif du droit des populations autochtones [...] pour le renforcement des capacités de gestion, d'organisation, de planification, d'administration et de commercialisation de la forêt pour générer un plus grand contrôle dans l'usage durable et diversifié des ressources avec une vision d'entreprise communautaire forestière »[FUNICA, Fundación Ford and CRAAN-GRAAN, 2010]. Dès le début des années 2000, des projets, promus et financés par des acteurs de développement, se multiplient, encouragés par la récente promulgation de la stratégie de développement forestière régionale de la Côte Atlantique en 2003 et l'inscription de la notion dans des documents de politique [FUNICA et al., 2010]. En 2008, la stratégie nationale forestière et le plan de développement de la Côte Caraïbe (2008-2012) définissent la foresterie communautaire comme un axe prioritaire pour promouvoir l'exploitation durable des forêts dans ou en périphérie d'aires protégées. Pour les populations locales, la foresterie communautaire peut être vue comme une aubaine pour mettre en pratique leurs droits récemment reconnus par l'État sur le foncier et les ressources, et pour affirmer l'importance de leurs modalités d'usage de la forêt qui sont en lien avec une cosmovision en harmonie avec la nature, garante d'un usage rationnel, intégral et équilibré des ressources.

Toutefois, deux décennies après le lancement de la foresterie communautaire dans la Côte Atlantique Nord du Nicaragua, les résultats sont mitigés : si des réussites techniques et organisationnelles sont indéniables, des problèmes importants demeurent. La situation écologique, économique et sociale dans les communautés autochtones reste précaire, marquée par la pauvreté et le sous-emploi, ainsi que par des conflits, parfois violents, à plusieurs niveaux et de plusieurs ordres. Il est central de rappeler que, par ailleurs, la forêt dégradée est menacée par l'extraction illégale du bois et par les défrichages continus de populations mestizas [Pérez Brignoli, 2003] - souvent pauvres et démunies - en quête de parcelles de culture ou de pâturages.

Dans le présent article, nous examinons le processus d'introduction et de réappropriation locale de la foresterie communautaire par les communautés autochtones de la Côte Atlantique Nord du Nicaragua. Nous analysons comment ces projets ont entraîné une crise profonde dans la gouvernance communautaire des ressources forestières qui, à l'origine, est fondée sur la cosmovision des usages, des pratiques et un système de normes qui en découle. En effet, en ignorant les formes d'usage des forêts des communautés autochtones et en introduisant de nouvelles règles de gestion forestière, les pratiques d'extraction de bois mises en œuvre dans les projets de foresterie communautaire se heurtent aux modes de fonctionnement spécifiques des institutions communautaires. Après avoir apporté des précisions méthodologiques sur la recherche conduite dans six communautés miskitas dans lesquelles nous avons analysé les institutions régissant l'exploitation des ressources forestières, notamment l'Assemblée communautaire, nous rappellerons comment la cosmovision, les usages et les pratiques locales autour de la forêt orientent la vie et définissent les institutions responsables des décisions 
communautaires sur la Côte Atlantique Nord. Puis nous présenterons les origines et la mise en place et l'évolution de la foresterie communautaire au Nicaragua. Nous verrons ainsi comment le projet de foresterie communautaire a bouleversé les institutions communautaires et exacerbé et aggravé les tensions au sein de la population, dues à l'accaparement des bénéfices de l'extraction forestière par une minorité. Nous conclurons sur des éléments de réflexion sur les perspectives de développement durable de ces régions forestières.

\section{Précisions méthodologiques}

Les résultats et l'analyse présentés dans la suite de cet article reposent sur des travaux réalisés dans le cadre d'un projet de recherche ${ }^{3}$ visant à cartographier et analyser les institutions gouvernant les ressources naturelles au Nicaragua et au Honduras [Fréguin-Gresh et al., 2014a, Fréguin-Gresh et al., 2014b]. L'approche théorique mobilisée s'inspire de l'analyse institutionnelle pour le développement (IAD) ${ }^{4}$ qui propose d'examiner les formes selon lesquelles les êtres humains interagissent avec la nature dans l'usage de «biens communs » et d'identifier les arrangements institutionnels permettant d'éviter l'épuisement de ces ressources [Ostrom, 2007].

L'analyse présentée dans cet article repose sur l'exploitation des entretiens ouverts et des groupes de discussions conduits au cours du deuxième semestre 2013 dans six communautés du territoire indigène de Twi Yahbra au Nicaragua (anciennement nommé Diez Comunidades), Sangnilaya, Iltara, Panua, Butku, Auhya Tara et Auhya Pihny (figure 1), qui forment ensemble un site connu sous un acronyme, SIPBAA, renvoyant aux premières lettres du nom de ces communautés. Les informations recueillies portent sur l'histoire, le peuplement et les caractéristiques des communautés autochtones du site, ainsi que sur l'évolution de l'usage et des règles d'accès et d'exploitation des ressources naturelles par ces populations. Un accent particulier a été porté sur les relations des populations avec les ressources naturelles, en particulier à travers la mobilisation des savoirs autochtones.

3. Le projet a reçu l'appui financier du Centre mondial de l'agroforesterie (ICRAF) dans le cadre de son programme de recherche «Forêts, arbres et agroforesterie : moyens d'existence, paysages et gouvernance » du Groupe consultatif de recherche internationale en agriculture (CGIAR), à travers son initiative Sentinel Landcape. Le projet a été conduit entre 2013 et 2014, sous la coordination du CIRAD. Il a été élaboré et mis en œuvre par le CIRAD, l'Institut de recherche et de développement Nitlapan et le Centre d'agriculture tropicale et d'éducation supérieure (CATIE). Le travail de terrain au Nicaragua a été supervisé par Sandrine Fréguin-Gresh et mis en œuvre avec Ceferino Wilson White, Pilar Elisa Müller Oporta, Alejandro Pikitle, Roberto Marchena Williams, Mike Philipp Müller.

4. À partir des instruments (questionnaires fermés, guides d'entretiens semi-structurés) développés par le programme de recherche sur les ressources et les institutions forestières (IFRI) issus du cadre IAD (Institutional analysis and development framework), nous avons élaboré des outils de collecte (guides d'entretiens ou de conduite de groupes de discussion, questionnaires) et recueilli des informations permettant d'étudier la morphologie spatiale et socio-économique d'un site d'étude, d'en comprendre les règles du jeu et d'examiner le rôle des acteurs dans cette gouvernance. 
Figure 1 - Localisation du territoire indigène de Twi Yahbra sur la Côte Atlantique Nord du Nicaragua
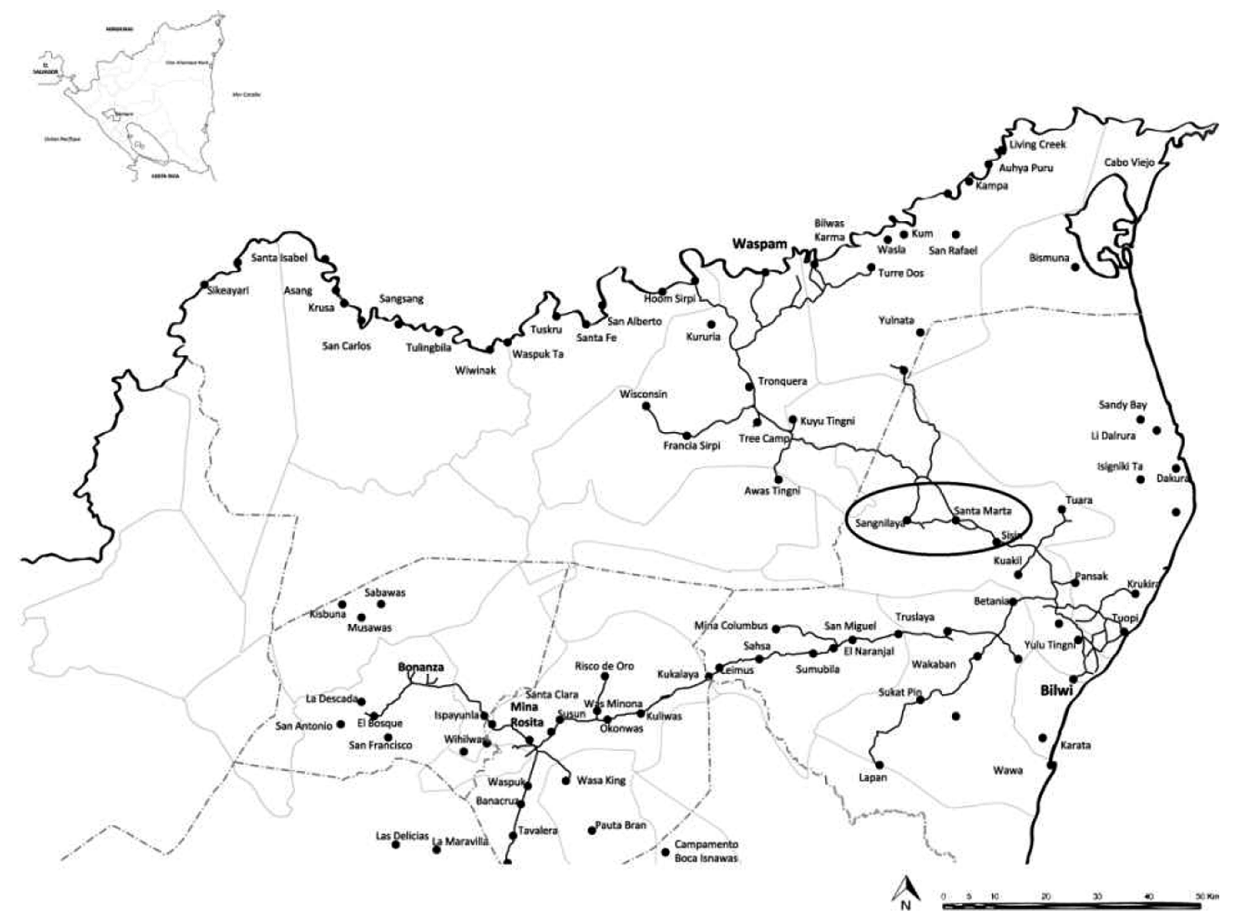

Source : auteur, d'après différentes cartes de l'INETER.

Nous avons choisi le site de SIPBAA, en raison de l'importance de la forêt dans les moyens d'existence des populations locales; du maintien de savoirs autochtones, ainsi que de normes et de pratiques communautaires en lien avec ces savoirs; de l'existence de plusieurs niveaux de prise de décision (national, régional, territorial, communal) qui se traduit localement par un système de normes spécifiques; de la présence de droits autochtones sur le foncier et les ressources naturelles, mais aussi de droits individuels et d'autres types de droits collectifs sur le foncier; de l'existence de tensions sur les ressources, en particulier à cause de la déforestation, de coupes illégales de bois et de l'occupation sans autorisation de terres par des populations allochtones qui défrichent la forêt; et enfin de la mise en œuvre d'un projet de foresterie communautaire. Les dynamiques en œuvre dans le site d'étude sont, pour cela, illustratives de celles en cours dans nombre de communautés autochtones de la Côte Atlantique Nord du Nicaragua.

\section{Populations et pratiques productives}

D'après les informations recueillies dans le projet, la population de SIPBAA compte près de 3500 personnes qui s'identifient Miskitas (90\%), Mestizas (9\%) ou d'autres ethnies (mayangnas et créoles). Cette population vit essentiellement 
de l'exploitation de deux écosystèmes (figure 2) : la plaine, aux sols latéritiques pauvres, qui est couverte d'une végétation éparse de pins caraïbes utilisés pour la construction de maisons, qui héberge de nombreux animaux chassés pour leur viande ; la forêt dense sempervirente tropicale humide, qui est composée d'espèces ligneuses diversifiées, qui a de multiples usages : elle est cultivée ${ }^{5}$, fournit du bois de feu, héberge des animaux (chassés, pêchés), des plantes (utilisées pour la magie, les rituels et la médecine traditionnelle) et produit du bois d'œuvre pouvant être commercialisé. Toutefois, un nombre réduit de personnes extrait et vend le bois : si l'extraction de bois à des fins commerciales s'opère dans 4 des 6 communautés, elle n'implique réellement qu'entre 2 et $20 \%$ des habitants des communautés. Si certaines personnes accèdent à des emplois salariés (éducation, santé, projets de développement) et pratiquent des activités non agricoles (artisanat, boulangerie, ébénisterie, vente de détail, extraction artisanale de minerais dans les rivières), la grande majorité tire ses moyens d'existence de la forêt.

Figure 2 - Carte de situation des communautés SIPBAA et de la zone d'exploitation forestière
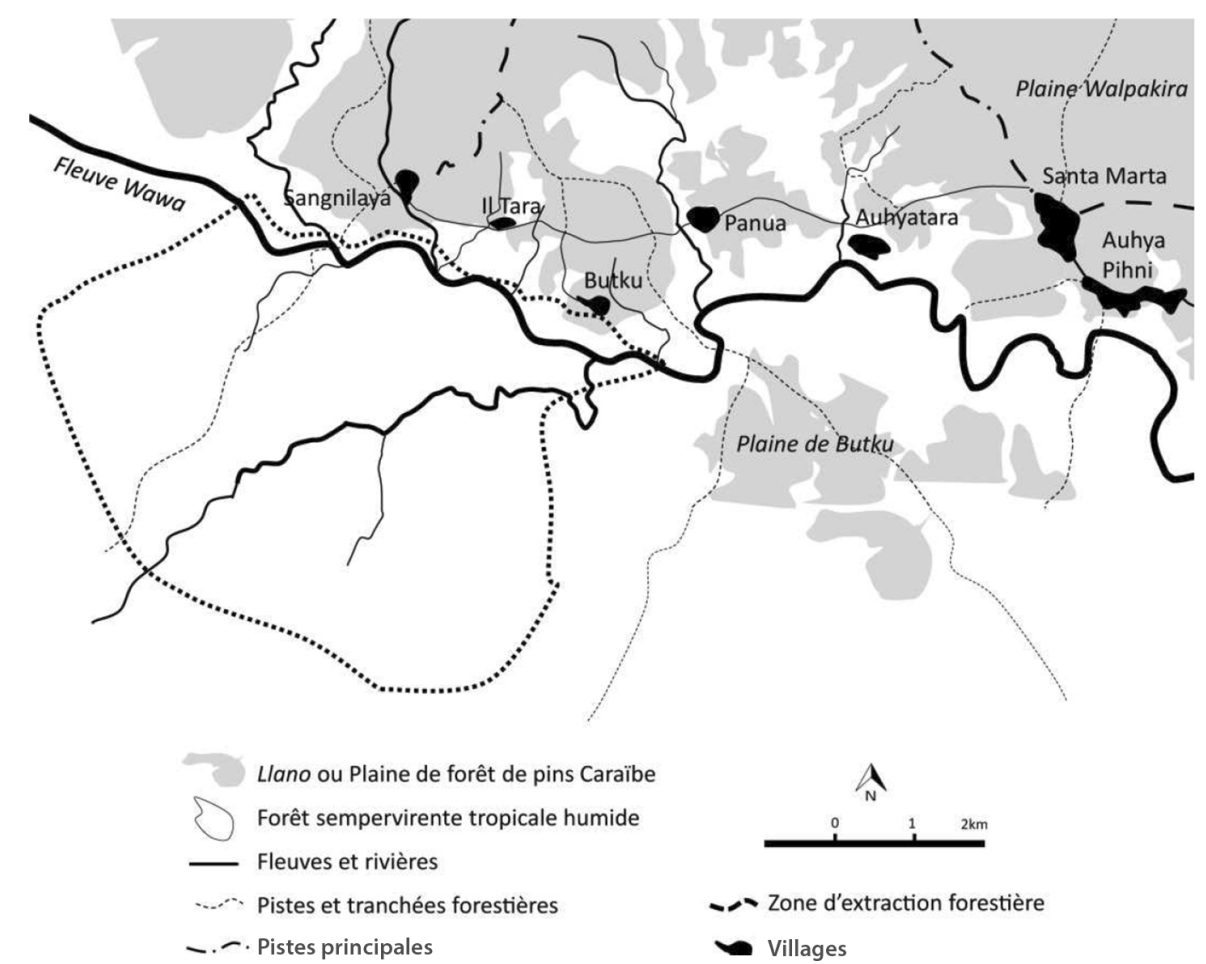

Sources : auteur, d'après des cartes topographiques $1: 50000^{\circ}$ et le plan général d'exploitation forestière.

5. Les populations y installent des champs de riz, haricots, racines, bananes plantains et tubercules sous les arbres forestiers selon des techniques ancestrales d'abattis-brûlis-pourrissage, basées sur leurs connaissances de l'écosystème. 


\section{Les savoirs autochtones comme cosmovision, pratiques productives et institutions régulant la vie des communautés de la Côte Atlantique}

La Côte Atlantique Nord du Nicaragua est un espace géographique à part entière : d'un point de vue politique, elle a intégré tardivement le reste de la nation à la fin du XIX ${ }^{e}$ siècle ${ }^{6}$ et bénéficie depuis la fin des années 1980 d'un statut d'autonomie qui lui confère un certain niveau de décentralisation, spécifique dans le pays ; d'un point de vue spatial, elle est restée enclavée, et malgré la pression accrue d'une colonisation agraire depuis le Pacifique et le centre du pays, elle est isolée de certaines dynamiques démo-économiques en œuvre ; d'un point de vue socioculturel enfin, la région se démarque de celles du Pacifique et du Centre, car les populations autochtones y ont conservé leurs savoirs, us et coutumes. Cette situation se traduit par une articulation de la région avec d'autres pays de la Caraïbe plus forte qu'avec ceux d'Amérique Centrale et est renforcée par les revendications identitaires ${ }^{7}$ et politiques des populations autochtones qui l'habitent [Gros, 1989].

La Côte Atlantique Nord du Nicaragua est peuplée de plusieurs groupes ethniques : Mestizos $^{8}$, Sumomayangna, Miskitu, Créoles, Garífunas et Ramas, qui cohabitent dans des conditions biophysiques spécifiques (zones de montagne, piémonts et plaines soumises à un climat tropical humide ayant donné lieu à des écosystèmes boisés, variables selon le sous-sol). Les Miskitus forment le groupe ethnique le plus nombreux, même si les estimations concernant leur nombre varient fortement, d'autant que leur auto-identification est avant tout politique ${ }^{9}$ [Roué, 2012]. Les Miskitus se concentrent sur un espace transfrontalier comprenant le nord-ouest du Nicaragua et le sud-ouest du Honduras (aussi appelé la Mosquitia). D'origine chibcha, ils se divisent en plusieurs sous-groupes ayant chacun leurs particularités : les Mam aux Honduras, les Wanky sur les rives du fleuve frontalier Coco, les Tawira de Sany Bay à Wauhta et les Baldam de Laguna de Perla [MASTA, 2012]. Il est admis que les Miskitus ont largement incorporé des populations d'autres ethnies autochtones, ou d'origines africaines et européennes qui ont, à un moment de l'histoire, fréquenté cet espace. Malgré les

6. La fin du Protectorat anglais est actée en 1894.

7. Essentiellement autochtones et dans une moindre mesure, afrodescendante.

8. «Mestizaje » en espagnol, qui donne lieu au terme «mestizo » que nous choisissons de garder en espagnol dans le texte vu qu'il renvoie à un concept différent du terme «métisse » en français.

9. Dès les années 1970 , la reconnaissance de l'occupation communale des terres pour les populations autochtones au Nicaragua devient le fer de lance d'un conflit politique, social et ethnique majeur opposant les populations autochtones avec l'État nicaraguayen. Dans cette lutte, la mobilisation de la cosmovision est centrale et aboutit à l'inclusion dans la Constitution d'éléments engageant à la reconnaissance de droits des peuples autochtones sur la terre et les ressources (art. 89). La loi sur le régime de propriété communale en 2003 rend effective et légale la propriété communale sur la Côte Atlantique. Puis, d'autres textes régulant l'exploitation des ressources naturelles reprennent ces éléments : la loi générale de l'environnement et des ressources naturelles ( ${ }^{\circ} 217$ de 1996 et sa réforme de 2008), la loi sur la conservation, la promotion et le développement durable du secteur forestier $\left(n^{\circ} 462\right.$ de 2003) qui posent les bases de la gestion des forêts naturelles, la promotion des plantations forestières et les bases pour la conservation et la restauration des espaces boisés ou encore la loi qui régule les coupes illégales et l'exploitation d'essences précieuses $\left(\mathrm{n}^{\circ} 585\right.$ de 2006). 
différences existant au sein des Miskitus, des éléments structuraux les rassemblent : le maintien de l'usage d'une langue, l'organisation de la parenté avec une résidence après le mariage dans la famille de la mariée. La force des relations de proximité de la famille étendue maternelle sont, entre autres, des caractéristiques de tous les Miskitus.

Les Miskitus ont par ailleurs conservé des savoirs autochtones basés sur la cosmovision [Brunelle, 2010] dont le principe repose sur le fait que les êtres humains doivent vivre dans une relation intégrale avec Mère Nature et ses éléments, auxquels ils appartiennent, mais qu'ils ne dominent pas [Cunninghan Kain, Mairena Aráuz, Pacheco Sebola, 2010]. La cosmovision imprègne les savoirs et dirige les pratiques culturelles et productives, comme les institutions qui les régulent. Cette cosmovision est basée sur la connaissance empirique du milieu environnant dont l'usage a été validé de génération en génération, à l'échelle locale. Par conséquent, les savoirs autochtones portés par ces populations peuvent être définis comme des : «ensembles cumulatifs et complexes de savoirs, savoir-faire, pratiques et représentations qui sont perpétués et développés par des personnes ayant une longue histoire d'interaction avec leur environnement naturel, ces systèmes cognitifs [faisant] partie d'un ensemble qui inclut la langue, l'attachement au lieu, la spiritualité et la vision du monde » [Unesco, sans date].

Toutefois, il serait naïf de croire que la relation que les Miskitus entretiennent avec la nature est systématiquement écologique et harmonieuse : il existe une forte hétérogénéité au sein de la population, fondée sur la différenciation socio-économique et des intérêts divergents relatifs à la gestion des ressources [Mairena Cunningham, 2007]. En effet, la population est hiérarchisée autour de figures de pouvoir qui assurent la transmission des savoirs et sanctionnent les personnes qui ne respectent pas les normes traditionnelles : les guérisseurs et les sages-femmes garantissent l'usage, la conservation et la transmission des connaissances sur les plantes utiles à des fins spirituelles et sanitaires, alors que les élus en assemblées communautaires et responsables de l'administration des ressources naturelles (wihtas et sindicos) veillent à l'application des règles, sous la bienveillance d'un conseil d'Anciens qui participent à la résolution des conflits et à la transmission des savoirs. Pourtant, alors que les wihtas et sindicos sont élus sur la base de la reconnaissance de leurs savoirs sur le milieu environnant, ainsi que sur leurs capacités et leurs expériences des règles, leurs conceptions de conservation et le respect des valeurs ancestrales [Cunninghan Kain et al., 2010], il est fréquent que l'exercice de leur fonction soit remis en cause et qu'ils soient destitués : des alliances avec des acteurs externes à la communauté (notamment des populations allochtones installées sans droit reconnu ou même après avoir versé des pots-de-vin) ou encore des soupçons de corruption (en particulier lors de la vente de bois précieux ou de versement d'aides internationales) ont marqué l'histoire des communautés miskitas. Aussi, depuis les années 1940, le développement de l'extraction de bois par des compagnies étrangères et la vente de bois se sont progressivement insérés dans les activités locales pour devenir une activité courante, bien que limitée [Mairena Cunningham, 2007]. Cette pratique, comme nous 
le verrons par la suite, a renforcé la différenciation socio-économique, scindant la population entre une minorité capable de générer des revenus monétaires par l'extraction et la vente de bois, et la majorité qui en est exclue. Ainsi, la population utilise la forêt tropicale humide pour l'agriculture et l'exploitation des espèces ligneuses et non ligneuses, combinées à d'autres activités (pêche, chasse, cueillette) qui lui permettent de satisfaire ses besoins. Ces pratiques se fondent sur des savoirs reconnus pour la conservation du milieu naturel : intégration de l'arbre forestier dans des systèmes de polyculture complexes, fondés sur l'abattis-brûlis-pourrissage sélectif de certaines espèces et sur une rotation entre la culture et la friche arborée de plusieurs décennies ; prélèvement sélectif et sur de grands espaces de ressources (plantes médicinales ou à vocation spirituelle, animaux sauvages pour la chasse et la pêche). Les Miskitus ne cultivent que de petites surfaces aux abords des cours d'eau et en pleine forêt à peine défrichée pour garantir l'alimentation de la famille tout en assurant la régénération du milieu. Si l'extraction d'espèces ligneuses est commune, sa pratique est réalisée pour obtenir des matériaux de construction à des fins domestiques ou communautaires (maisons, pirogues, église, etc.) et non pour générer des profits commerciaux [MASTA, 2012].

\section{Les règles de gestion « traditionnelle » des ressources naturelles : des institutions établies sur la base des savoirs autochtones}

La gouvernance, c'est-à-dire le système de règles en usage localement, repose sur une combinaison de réglementations élaborées par le gouvernement au plan national et de normes orales locales : la Constitution, les instances politiques et la législation nationale et régionale pour l'accès, les usages et la gestion des ressources naturelles, ainsi que des règles communautaires [Fréguin-Gresh et al., 2013]. Ces règles, formelles ou non, écrites ou non, intéressantes pour la communauté, sont interprétées par les élus (wihtas et sindicos) et votées en assemblées communales. Elles déterminent pour chaque type de ressource naturelle une valeur d'usage, de marché ou de conservation.

À SIPBAA, où les ressources naturelles relèvent du domaine de chaque communauté, la norme régissant la coupe des arbres dépend de l'espèce, de sa localisation et de sa destination, à savoir la conservation ou l'abattage « raisonné » : il est possible de couper sans autorisation spécifique un pin caraibe dans la plaine, car il est admis que sa régénération naturelle est rapide et que l'usage du bois est destiné à des fins domestiques ou communautaires pour la construction des maisons sur pilotis ; en revanche, il faut obtenir une autorisation et payer une taxe auprès du sindico pour abattre une espèce ligneuse «précieuse » de la forêt sempervirente (cèdre, acajou), si tant est que l'arbre ne soit pas un arbre « mère » (c'est-à-dire fournisseur de semences permettant la régénération), car les Miskitus en limitent l'extraction pour permettre à la forêt de se régénérer.

Dans l'espace communautaire, pour limiter la surexploitation de la forêt ou des berges cultivables, tout en permettant aux familles natives et leurs alliés par 
le mariage (les seules pouvant théoriquement bénéficier des droits communaux) d'assurer la couverture de leurs besoins, les règles gouvernant l'accès au foncier (et aux ressources qui s'y trouvent) sont précises : une famille n'est autorisée à défricher une parcelle que si celle-ci n'est pas en possession d'une autre famille et est située dans un espace qui n'est pas déjà saturé (pour permettre le renouvellement de la fertilité par la régénération naturelle ou les crues). L'assignation de parcelles se déroule en plusieurs étapes : l'identification d'un espace libre et favorable à la mise en culture ; la demande auprès des représentants élus de la communauté ; le vote et la décision de l'assignation en assemblée, actée par le wihta, et la mise à disposition de la famille ${ }^{10}$ pour l'agriculture ou l'extraction pendant une durée indéterminée. Sur les parcelles attribuées, les membres d'une famille s'organisent entre eux pour que chacun, homme et femme, accède à ce dont il ou elle a besoin : cultiver, chasser, collecter et extraire le bois, selon les règles communautaires établies, parfois contestées par la population.

\section{La foresterie communautaire dans les communautés miskitas de SIPBAA : introduction, mise en œuvre et défis}

\section{La création d'une organisation de base communautaire, aux compétences "renforcées ", dont le fonctionnement remet en cause des pratiques fondées sur les savoirs autochtones}

Dans le contexte d'émergence de projets de foresterie communautaire dans la Côte Atlantique Nord, les représentants élus des communautés SIPBAA décident de créer en 2002 une association de base communautaire. Cette association cherche à bénéficier du projet pour les forêts (PROFOR, de la Banque mondiale) et de ceux du Fonds mondial pour la nature (WWF) visant à promouvoir la commercialisation de bois certifié et l'accès au marché du bois des communautés autochtones [Romero J. et al., 2010] par son programme SmartWood et l'appui du Forest Stewardship Council, un organisme promouvant la certification de forêts gérées socialement et écologiquement. L'association se forme autour d'un conseil d'administration dont les membres sont choisis en assemblées communautaires. Leur condition d'éligibilité repose sur l'origine (être natif) et la représentativité (en termes de niveau d'éducation et socio-économique, d'ethnie, de partis politiques). Les membres du conseil d'administration sont naturellement les représentants élus (wihtas et sindicos), les porte-parole légitimes des communautés au moment de la création de l'association.

Entre 2003 et 2005, WWF finance le montage d'un projet de foresterie communautaire à SIPBAA. WWF s'appuie alors sur l'association communautaire créée. Le projet suit plusieurs objectifs : (i) l'extraction forestière par les communautés, sous le contrôle de l'association de base et selon un modèle entrepreneurial ; (ii) la technicisation des pratiques d'extraction respectant des normes respectueuses

10. Ces droits ne concernent que l'exploitation, car ni la terre ni les ressources qui s'y trouvent, ne peuvent être cédées, c'est-à-dire, ne peuvent être vendues, louées ou hypothéquées de manière légale. 
de fl'environnement, et permettant la certification ; (iii) la mise en place de plans de gestion stratégiques et d'investissement, pour développer les infrastructures et les services dans les communautés grâce aux bénéfices de la vente de bois ; (iv) le renforcement des compétences locales en management. L'association communautaire s'engage à s'appuyer sur une entreprise locale de transformation du bois qui, en retour, fournit des formations techniques aux membres de l'association.

En 2005, l'association obtient le statut de coopérative agroforestière, nommée COOSIPBAA (Coopérative des communautés de SIPBAA). Cette formalisation lui permet d'accéder à d'autres financements de la coopération (coopération bilatérale allemande, hollandaise, norvégienne, etc.), à des formations professionnelles en coopérativisme et en gestion entrepreneuriale, qui sont obligatoires au plan national (loi sur les coopératives). Au total, treize personnes sont formées en 2005 , dix en 2010 [von Marschalck, 2012]. À l'heure actuelle, ces 23 personnes forment le groupe des associés de la coopérative et la gèrent avec des appuis et de l'assistance technique étrangers : par exemple, les techniciens forestiers de WWF encadrent les associés de COOSIPBAA pour délimiter les espaces où les coupes d'arbres sont à réaliser et supervisent les coupes.

L'espace délimité pour les coupes est de 77500 ha (figure 2). Sa taille considérable devient rapidement sujette à de nouvelles règles : un plan général d'extraction forestière, un plan d'investissements et des plans d'opération annuels, tous élaborés par le conseil d'administration de COOSIPBAA en collaboration avec l'assistance technique étrangère. Le plan général d'extraction prévoit la contractualisation de personnel local pour réaliser l'abattage et la préparation des arbres, en vue de leur transformation par une entreprise régionale: Masangni [Matamoros, 2007]. Mais la population est peu consultée dans les prises de décisions de cette organisation d'exploitation de la forêt, même si l'aval des assemblées communales est acquis.

COOSIPBAA semble alors très éloignée des intérêts que l'association communautaire défendait à l'origine. Et les renforcements de compétences techniques des associés, la mise en place d'un système de gouvernance en collaboration uniquement avec les techniciens de WWF, sans consultation des règles et modalités d'usage communautaires des ressources forestières, ont rapidement posé des problèmes au sein des communautés autochtones. Notre analyse fait écho, par de nombreux aspects, à d'autres constats des défis de la foresterie communautaire en Afrique [Cuny, 2011].

Le premier point de blocage, qui est un élément essentiel contraignant du projet de foresterie communautaire, repose sur l'insuffisante compréhension de la notion de communauté par les organisations étrangères (WWF notamment) dans le contexte des populations Miskitus de la Côte Atlantique Nord. En effet, comme nous l'avons déjà mentionné, les communautés sont hétérogènes. Certains auteurs [Mairena Cunningham, 2007] rappellent que des «élites » socio-économiques, éduquées et issues des familles mieux loties, car bénéficiant de revenus extérieurs, s'imposent facilement comme représentants élus des communautés : accédant à la 
fois aux sphères de pouvoir légitimes et de décision avec COOSIPBAA, ces élus deviennent irremplaçables et le fonctionnement de la coopérative dépend d'eux, de manière nominative. Or, le système de gouvernance traditionnelle est accepté de tous, car il est flexible et permet de destituer par vote en assemblée communale des représentants élus, dès lors que la population n'adhère plus à leurs actions et décisions. C'est ce qui s'est passé à SIPBAA quand, soupçonnés de malversations et de détournement de fonds, les associés, représentants élus au moment de la création de l'association, siégeant de facto de manière permanente au conseil d'administration, ont été destitués comme représentants légitimes, mais sont restés associés de la coopérative.

Une deuxième difficulté repose sur le manque de participation et d'adhésion de la population supposée être engagée dans l'exploitation forestière. Le projet est centré sur l'extraction commerciale de bois (malgré le nom de la coopérative, définie comme «agroforestière », mais qui n'en a que le nom). Étant donné que les pratiques productives de la grande majorité sont centrées sur l'agriculture combinée à d'autres usages des ressources forestières et non sur l'extraction commerciale du bois [Offen, 2002], la population se désintéresse progressivement des activités de COOSIPBAA. Ces dernières restent d'ailleurs hors de portée d'une grande part de la population (absence de matériel ou de savoir-faire dans l'abattage d'arbres de forêt tropicale dense ou dans la transformation du bois). Aussi, la coopérative ne s'investissant pas dans ses moyens d'existence, l'agroforesterie, la population ignore tout simplement l'existence de COOSIPBAA. De plus, les associés ne percevant aucun salaire pour leur engagement et étant accusés de détourner les ressources tirées de l'exploitation commerciale du bois à leurs profits, ils se démotivent dans leur participation aux instances communautaires et préfèrent s'adonner aux activités leur permettant d'assurer leur subsistance. L'introduction du salariat dans les communautés pour l'extraction forestière génère aussi des tensions entre ceux qui sont rémunérés et ceux et qui ne le sont pas; ces derniers étant les plus nombreux.

Un troisième défi renvoie directement aux fondements des pratiques productives et des règles de gestion participative des forêts au moment des entretiens. Selon les élus des communautés, la loi nationale des coopératives contredit la loi foncière sur le régime communal. Le plan général d'exploitation de COOSIPBAA autorise les associés à décider des modalités d'extraction du bois (qui, pour des raisons logistiques, exclut la population de l'octroi de parcelles dans les aires destinées aux activités extractives). Or, la population ressent une usurpation de ses droits et ne comprend pas comment les associés, un petit noyau de personnes sans légitimité peuvent décider de l'usage de « leur» forêt pour en tirer des profits « pour la coopérative ».

D'un point de vue écologique, la population considère que l'approche technique «moderne » promue par WWF et réappropriée par COOSIPBAA dans les plans de gestion ne prend pas en compte leurs savoirs. Cette extraction, présentée pourtant comme respectueuse de l'environnement ou écologique, détériore la forêt, 
alors que celle-ci est déjà menacée par l'exploitation illégale du bois et par les défrichages continus de populations mestizas en quête de parcelles de culture ou de pâturages.

\section{Le manque de transparence et l'invisibilisation des avantages de l'extraction forestière pour le plus grand nombre : une menace pour la durabilité}

Dès le lancement des opérations de COOSIPBAA, d'autres difficultés apparaissent. En 2005, la vente de bois permet de creuser une tranchée permettant d'acheminer les arbres abattus hors de la forêt ; cette tranchée passe en partie dans les terres d'une communauté voisine dont les représentants communaux bloquent la sortie du bois en signe de protestation, provoquant des pertes et amoindrissant les bénéfices générés. En 2006, la promulgation de la loi de fermeture saisonnière de l'extraction forestière empêche le bon déroulement des activités et entraîne encore des pertes, partiellement couvertes par les bénéfices des ventes de la première année. En 2007, l'ouragan Felix dévaste une bonne partie des forêts de la Côte Atlantique Nord, ce qui entraîne l'annulation du plan d'opération annuel en faveur d'un plan de sauvetage du bois tombé par l'ouragan ${ }^{11}$; le bois extrait est utilisé pour la reconstruction de maisons pour les membres des communautés touchés par la catastrophe. Cette opération, si elle ne génère pas de profits, est particulièrement bien accueillie par la population qui voit pour la première fois des résultats concrets de l'activité forestière. Il n'y a pas d'extraction en 2008 et 2009, pour diverses raisons ; mais la coopérative reçoit des dons et de l'aide alimentaire à la suite de l'ouragan. En 2010 et 2011, de nouvelles coupes de bois ont lieu; les bénéfices sont utilisés pour payer des salaires et ouvrir une nouvelle tranchée, une partie importante permet de couvrir les coûts de la certification. En 2012 et 2013, des extractions ont lieu dans un climat de conflits au sein du conseil d'administration suspecté de corruption et entre celui-ci et le reste de la population qui lui réclame plus de transparence.

Rares sont les habitants des communautés qui voient dans l'exploitation forestière durable un bénéfice collectif. Même s'il existe un conseil de surveillance de la coopérative, constitué des wihtas et síndicos actuels, il semble que le mécanisme de contrôle social, à la base de la consultation de la population et créé en même temps que la coopérative soit déficient, voire inactif. Pour ces raisons, les nouveaux représentants communautaires ont choisi en 2014 de passer un accord directement avec une compagnie étrangère pour exploiter le bois, court-circuitant COOSIPBAA. Aujourd'hui, la coopérative est perçue comme étrangère aux communautés, exploitant une forêt sans le soutien de la population qui n'en voit aucun bénéfice.

11. Ce décret fût largement décrié et entraîna l'augmentation considérable des abattages illégaux d'arbres. 


\section{Conclusions et perspectives}

La foresterie communautaire s'est imposée au Nicaragua comme dans d'autres pays, mettant en avant la nécessité d'inclure les populations vivant dans, à proximité des forêts tropicales dans leur gestion et leur exploitation. Elle s'appuie sur une philosophie selon laquelle, en respectant les savoirs autochtones, l'exploitation de la forêt est durable, c'est-à-dire qu'elle apporte des bénéfices sociaux et économiques aux populations, tout en conservant les ressources. Ainsi, sur la Côte Atlantique Nord du Nicaragua, la foresterie communautaire s'est développée dans des communautés autochtones où les populations revendiquent depuis longtemps des pratiques basées sur des usages des ressources forestières en rapport avec des règles régies par leurs assemblées communautaires. Cependant, telle qu'introduite dans certaines communautés indigènes, la foresterie communautaire n'a pas apporté les résultats espérés. Si ces projets affichent la prise en compte de savoirs autochtones et de normes traditionnelles dans leur conception, leur mise en œuvre réelle est toute autre : avec des pratiques techniques industrielles et mécanisées d'extraction forestière massive, elle ne respecte pas les normes de gouvernance communautaire, indissociables des savoirs autochtones. Ainsi, dans les communautés SIPBAA, la foresterie communautaire n'a pas tenu ses promesses, puisque les ressources forestières continuent d'être menacées par la dégradation due au déboisement et aux occupations illégales.

Notre étude de cas amène alors à nous interroger sur l'ambigüité des rôles des acteurs de la coopération technique dans leurs interventions dans les zones forestières de la Côte Atlantique. En premier lieu, elle montre le déphasage entre les interventions et les besoins réels des populations : les conceptions et objectifs du projet de foresterie communautaire ont été élaborés sans la participation des populations locales, comme le montre l'absence d'une dimension agricole dans le projet. Si les modes de gouvernance participative basés sur l'assemblée communautaire ont été associés à certaines étapes du projet, leur centrage sur l'extraction commerciale de bois met en doute leur adéquation avec les aspirations et les conditions de vie des populations qui n'aspirent pas, pour la majorité, à s'engager dans l'abattage massif d'arbres et la vente de bois. De plus, le modèle de gestion choisi pour ce projet, fondé sur un modèle de coopérativisme est basé uniquement sur des aides financière et technique externes. Il aboutit alors à une sorte de privatisation de la forêt communautaire qui bénéficie in fine à une poignée d'anciens représentants formés par l'assistance technique et bien sûr, aux entreprises exportatrices de bois. Ainsi, si la recherche d'une solution aux problèmes locaux se base uniquement sur la question de la génération de revenus localement, et ce, seulement par l'exploitation des forêts, la foresterie communautaire telle qu'elle a été mise en œuvre n'est pas socialement acceptée, car ni équitable ni durable, elle génère de nombreux conflits. Ces derniers s'ajoutent aux tensions préexistantes exacerbées par les occupations et la déforestation illégales dans les espaces boisés reculés peu ou mal contrôlés par les communautés autochtones. Si aucune solution n'est trouvée, c'est la survie de ces populations dépendantes des forêts pour leurs multiples usages qui est menacée. Les risques encourus peuvent 
conduire à terme à l'épuisement des ressources forestières, qui sont pourtant au fondement de la vie des populations autochtones de l'Atlantique Nord du Nicaragua.

\section{Bibliographie}

ARnold J. E. M. [1991], Foresterie communautaire. Un examen de dix ans d'activité, Rome, FAO, Département des forêts.

Brunelle D. [2010], Gouvernance. Théories et pratiques, Montréal, Institut d'études internationales de Montréal.

Cunninghan Kain M., Mairena Aráuz D., Pacheco Sebola M. [2010], « Cambio climático : medidas de adaptación en comunidades de las regiones autónomas de la Costa Caribe de Nicaragua », Cuaderno de investigacion, $\mathrm{n}^{\circ} 82,51 \mathrm{p}$.

Cuny P. [2011], État des lieux de la foresterie communautaire et communale au Cameroun, Wageningen, Tropenbos international programme du bassin du Congo.

Fréguin-Gresh S., Müller Oporta P. E., Wilson White C., Flores lopéz J. C., Manzanares D., Huybrechs F., Pérez F. J. [2013], Regulations on access and property rights to natural resources in Nicaragua and Honduras: literature review for institutional mapping of the N-H Sentinel Landscape, Managua, CirAd, ICRAF, CATIE, Université de l'Amérique centrale (UCA)-Nitlapan.

Fréguin-Gresh S., Müller Oporta P. E., Flores Cruz S., Wilson White C. [2014a], Retos y desafíos de la forestería comunitaria en comunidades miskitus de la Costa Atlántica, VIII Congreso inter-disciplinario de investigación de la UCA, Université de l'Amérique centrale (UCA), Managua.

Fréguin-Gresh S., Wilson White C., Flores López J. C., Müller Oporta P. E, Huybrechs F., Pikitle A., Marchena Williams R., Manzanares D. [2014b], Mapping institutions that govern access and uses of natural resources in the Nicaragua-Honduras sentinel landscape : revealing the complexity, issues, and challenges of natural resource governance, Managua, CIRAD, ICRAF, CATIE, Université de l'Amérique Central (UCA)-Nitlapan.

FUNDACIÓN PARA EL DESARROLLO TECNOLÓGICO AGROPECUARIO Y FORESTAL DE NICARAGUA (Funica), Fundación Ford, Consejo y Gobierno Regional Autónomo del Atlántico NORTE (CRAAN-GRAAN) [2010], Aporte a la estrategía de forestería comunitaria en la RAAN, Informe técnico, Bilwi, FunICA, Fundación Ford, CRAAN-GRAAN.

Gros C. [1989], Foreterie communautaire au Nicaragua Nicaragua : un projet d'autonomie pour la Côte Atlantique. De la question régionale à la problématique ethnique », in ReVELMouroz J. (dir.), Pouvoir local, régionalismes, décentralisation, Paris, IHEAL, p. 459-470.

JARQUín M. J. [2003], Reforma institucional en Nicaragua : un reto permanente, VIII congreso internacional del CLAD sobre la reforma del Estado y de la Administración pública, Panama city, 28-31 octobre 2003.

Kaimowitz D., Faune A., Mendoza R. [2003], Your biosphere is my backyard: the story of Bosawas in Nicaragua, working paper $\mathrm{n}^{\circ} 25$, avril 2003, Jakarta, CIFOR.

LARSON A. M. [1989], «The Nicaraguan environment... A Legacy of destruction», Envio, $\mathrm{n}^{\circ} 100$ : www.envio.org.ni/articulo/2756 (consulté le 12 décembre 2016). 
LARSON A. M. [2002], « Natural resources and decentralization in Nicaragua : are local government up to the job ? », World Development, $\mathrm{n}^{\circ} 30$, p. 17-31.

Le Coq J.-F., Freguin-Gresh S., Saenz Segura F., Perez F. J. [2013], « Transfert de la notion de développement durable dans les politiques publiques centroaméricaines : lecture croisée des évolutions de référentiels et des trajectoires de politiques rurales au Costa Rica et au Nicaragua », colloque international CANAL 2013, Circulations et appropriations des normes et des modèles de l'action locale, Montpellier, 20-23 mars 2013.

Mairena Cunningham E. [2007], Gestión de los recursos naturales en comunidades indígenas de la Costa Caribe de Nicaragua, Managua, Université de l'Amérique centrale (UCA)-Nitlapan.

Marschalck A. (von) [2012], Análisis de la EFC COOSIPBAA y el Bloque de comunidades SIPBAA con enfoque en la toma de decisiones para desarrollar en conjunto un mecanismo para la resolución de conflictos, Managua, GIZ, Masangni.

Matamoros N. [2007], Plan de inversión 2007-2009, Cooperativa de producción agroforestal «SIPBAA », Generado de las utilidades de las operaciones forestales unidad de manejo "SIPBAA », Bilwi, Nicaragua, Consejo de administración de la cooperativa de producción agroforestal SIPBAA, Managua, Masangni, IFC (World Bank).

Milol C. A. [2007], "Gouvernance et participation dans la gestion des ressources forestières au Cameroun : impacts inattendus sur les pratiques foncières », in EBERHARD Ch. (dir.), Enjeux fonciers et environnementaux. Dialogues afroindiens, Pondichéry, Institut français de Pondichéry, p. 233-255.

Mosquitia asla takanKa-unidad de la mosquitia (Masta) [2012], Protocolo bio-cultural del pueblo indígena Miskitu. El derecho al consentimiento libre, previo e informado en nuestro territorio de La Muskitia Hondureña, Union international para la conservación de la naturalisa (UICN).

OFFEN K. [2002], « Ecología cultural miskita en los años 1650-1850 », Wani, n 30, p. 42-59.

Ostrom E. [2007], Institutional analysis and development : elements of the framework in historical perspective, Historial developments and theorical approaches in sociologie, Oxford, Encyclopedia of life support systems (EOLSS).

Pérez Brignoli H. [2003], La diversidad cultural y las lógicas del mestizaje en América Central, San José, universidad de Costa Rica, universidad nacional.

Roue M. [2012], « Histoire et épistémologie des savoirs locaux et autochtones. De la tradition à la mode », Revue d'ethnoécologie, $\mathrm{n}^{\circ} 1$, p. 2-14.

Sabogal C., De Jong W., Pokorny B., Louman B. [2008], Manejo forestal comunitario en América Latina. Experiencias, lecciones aprendidas y retos para el futuro, Bogor, CIFOR CATIE.

Organisation des Nations Unies POUR L'ÉDUCATION, LA SCIENCE ET La CULTURE (UNESCO) [sans date] : Systèmes de savoirs locaux et autochtones :www.unesco.org/new/fr/natural-sciences/ priority-areas/links/related-information/what-is-local-and-indigenous-knowledge/\#c 174259 (consulté le 12 décembre 2016).

UNITED STATUES AGENCE INTERNATIONAL DEVELOPPEMENT (UsAID) [2013], Les enseignements tirés de la foresterie communautaire en Afrique et leur pertinence pour REDD-PLUS, progamme carbone forestier, marchés et communautés (FCMC), Burlington (Vt), Agence des États-Unis pour le développement international.

Welbin Romero J., Perez F. J., Gallosi R, Gema Lorio L. [2010], Madera-muebles en Centroamerica : una posibilidad aun por aprovechar, Managua, Nitlapan. 